\title{
Dual spaces of multi-parameter martingale Hardy spaces
}

\author{
Ferenc Weisz * \\ Department of Numerical Analysis, Eötvös L. University \\ H-1117 Budapest, Pázmány P. sétány 1/C., Hungary \\ e-mail: weisz@inf.elte.hu
}

\begin{abstract}
In this paper we introduce the generalized multi-parameter martingale $B M O$ spaces. The atomic decomposition of the multi-parameter martingale HardyLorentz space $H_{p, q}^{s}$ is given. With the help of this, the dual space of $H_{p, q}^{s}$ is characterized as the generalized BMO space. Finally, as an application, JohnNirenberg inequality is generalized for multi-parameters.

Key words and phrases: Martingale Hardy-Lorentz spaces, multi-parameter martingales, generalized $B M O$ spaces, atomic decomposition, John-Nirenberg inequality.
\end{abstract}

2000 AMS Mathematics Subject Classifications: Primary 60G42, Secondary 60G46, 46E30, 60G48.

\section{Introduction}

Martingale Hardy-Lorentz spaces $H_{p, q}^{S}$ and $H_{p, q}^{s}$ defined by the quadratic and conditional quadratic variation are considered. The atomic decomposition is a useful characterization of martingale Hardy spaces by the help of which some duality theorems, interpolation results and martingale inequalities can be proved. The atomic decompositions of five different martingale Hardy spaces (amongst others the one of $H_{p}^{s}$ ) were given in [10]. More recently, using an idea of Abu-Shammala and Torchinsky [1], Jiao, Xie and Zhou [7] have extended the atomic decomposition to the martingale Hardy-Lorentz spaces $H_{p, q}^{s}$ (see also Jiao, Peng and Liu [6] and Ho [5]).

Multi-parameter martingales were investigated in several papers, see [10] and the references therein and they can be well applied in Fourier analysis (see [11]). The proofs for multi-parameter martingales are usually not simple adaptations of that of the one-parameter proofs. They need new ideas. The atomic decomposition for multiparameter martingale Hardy space $H_{p}^{s}$ is more complicated and is due to the author

*This research was supported by the Hungarian Scientific Research Funds (OTKA) No K115804. 
[10]. In this paper we generalize the preceding result of Jiao, Xie and Zhou [7] and characterize the atomic decomposition of the multi-parameter $H_{p, q}^{s}$.

A very classical result in harmonic analysis is that the dual of $H_{1}$ is $B M O$ (Fefferman and Stein [2]). For martingale Hardy spaces see Garsia [3], Long [8] and Weisz [10]. In [10] we proved that the dual of $H_{p}^{s}$ is $B M O_{2}(\alpha)$ if $0<p \leq 1, \alpha=1 / p-1$ and the dual of $H_{p}^{s}$ is $H_{p^{\prime}}^{s}$ if $1<p<\infty, 1 / p+1 / p^{\prime}=1$. The same holds also for multiparameter Hardy spaces (see [10]). Note that the situation is very different from the duals of the $L_{p}$ spaces if $0<p<1$, because the dual of $L_{p}$ is trivial. More recently, in the one-parameter case, Ho [5] characterized the dual of $H_{p, q}^{s}$ as the space $B M O_{2}(\alpha)$ when $0<p \leq 1,0<q \leq p, \alpha=1 / p-1$. Later Jiao, Xie and Zhou [7] and Ren [9] have generalized this result for all $0<p \leq 2,0<q \leq 1$. They [7] have introduced generalized $B M O_{2, q}(\alpha)$ spaces and have proved that the dual of $H_{p, q}^{s}$ is $B M O_{2, q}(\alpha)$ if $0<p \leq 2,1<q<\infty, \alpha=1 / p-1$. As a consequence, they [7] obtained a generalization of John-Nirenberg inequality, more exactly, $B M O_{2}(\alpha)$ is equivalent to $B M O_{r}(\alpha)$ and $B M O_{2, q}(\alpha)$ is equivalent to $B M O_{r, q}(\alpha)(1 \leq r<\infty)$. In this paper we generalize these results to multi-parameter Hardy-Lorentz spaces and generalized $B M O$ spaces.

\section{Martingales and dyadic Hardy spaces}

For a set $\mathbb{X} \neq \emptyset$ let $\mathbb{X}^{d}$ be its Cartesian product $\mathbb{X} \times \ldots \times \mathbb{X}$ taken with itself d-times. Let $d \geq 1$ be a fixed integer and let us introduce the following partial ordering on $\mathbb{N}^{d}$. For $n=\left(n_{1}, \ldots, n_{d}\right), m=\left(m_{1}, \ldots, m_{d}\right) \in \mathbb{N}^{d}$ set $n \leq m$ if $n_{j} \leq m_{j}$ for all $j=1, \ldots, d$. We say that $n<m$ if $n \leq m$ and $n \neq m$. Moreover, $n \ll m$ means that the inequalities $n_{j}<m_{j}$ hold for all $j=1, \ldots, d$. For $n=\left(n_{1}, \ldots, n_{d}\right)$ let $n-1=\left(n_{1}-1, \ldots, n_{d}-1\right)$.

For two arbitrary sets $H, G \subset \mathbb{N}^{d}$ consisting of incomparable number pairs (i.e. if $n, m \in H$ (or $G$ ) then neither of the inequalities $n \leq m$ and $m \leq n$ hold) we write $H \ll G$ (resp. $H \leq G$ ) if for all $n \in G$ there exists $m \in H$, such that $m \ll n$ (resp. $m \leq n)$. Denote by inf $H$ the set of the number pairs $m \in H$ for which there does not exist any $n \in H, n \neq m$ such that $n \leq m$. We shall use the convention $\inf \emptyset=\infty$.

Let $(\Omega, \mathcal{A}, P)$ be a probability space and let $\mathcal{F}=\left(\mathcal{F}_{n}, n \in \mathbb{N}^{d}\right)$ be a non-decreasing sequence of $\sigma$-algebras with respect to the partial ordering on $\mathbb{N}^{d}$. The expectation operator and the conditional expectation operator relative to $\mathcal{F}_{n}$ are denoted by $E$ and $E_{n}$. We suppose that

$$
E_{n}(f g)=E_{n} f E_{n} g \quad\left(n \in \mathbb{N}^{d}\right)
$$

for all bounded $\cup_{n_{i} \in \mathbb{N}, i \neq k} \mathcal{F}_{n}$-measurable functions $f$ and all $\cup_{n_{i} \in \mathbb{N}, i \neq l} \mathcal{F}_{n}$-measurable functions $g(k \neq l)$.

An integrable sequence $f=\left(f_{n}, n \in \mathbb{N}^{d}\right)$ is said to be a martingale if $f_{n}$ is $\mathcal{F}_{n}$ measurable $\left(n \in \mathbb{N}^{d}\right)$ and $E_{n} f_{m}=f_{n}$ for all $n \leq m$. For simplicity, we always suppose that for a martingale $f$ we have $f_{n}=0$ if $n_{1} \cdots n_{d}=0$. The stochastic basis $\mathcal{F}$ is said to be regular if there exists a number $R>0$ such that

$$
f_{n} \leq R f_{n_{1}-\epsilon_{1}, \ldots, n_{d}-\epsilon_{d}} \quad\left(n \in \mathbb{N}^{d}\right)
$$

holds for all non-negative martingales $\left(f_{n}, n \in \mathbb{N}^{d}\right)$ and all numbers $\epsilon_{j} \in\{0,1\}$ with $\epsilon_{1}+\cdots+\epsilon_{d}=1$. 
We briefly write $L_{p}$ instead of the $L_{p}(\Omega, \mathcal{A}, P)$ space while the norm (or quasinorm) of this space is defined by $\|f\|_{p}:=\left(E|f|^{p}\right)^{1 / p}(0<p \leq \infty)$. For a measurable function $f$, the non-increasing rearrangement is defined by

$$
\tilde{f}(t):=\inf \{\rho: P(|f|>\rho) \leq t\} .
$$

A measurable function is in the Lorentz space $L_{p, q}=L_{p, q}(\Omega, \mathcal{A}, P)(0<p<\infty, 0<$ $q \leq \infty)$ if

$$
\begin{aligned}
\|f\|_{p, q} & :=\left(\frac{q}{p} \int_{0}^{\infty} t^{q / p} \tilde{f}(t)^{q} \frac{d t}{t}\right)^{1 / q} \quad(0<q \leq \infty), \\
\|f\|_{p, \infty} & :=\sup _{t>0} t^{1 / p} \tilde{f}(t) \quad(q=\infty) .
\end{aligned}
$$

It is known (see Grafakos [4]) that

$$
\begin{aligned}
\|f\|_{p, q} & =\left(q \int_{0}^{\infty} t^{q} P(|f|>t)^{q / p} \frac{d t}{t}\right)^{1 / q} \quad(0<q \leq \infty), \\
\|f\|_{p, \infty} & =\sup _{t>0} t P(|f|>t)^{1 / p} \quad(q=\infty) .
\end{aligned}
$$

We recall that $L_{p, p}=L_{p}$ and $L_{p, q}$ increase as the second exponent $q$ increases, and decrease as the first exponent $p$ increases.

The quadratic variation and the conditional quadratic variation of a martingale $f=\left(f_{n}, n \in \mathbb{N}^{d}\right)$ are defined by

$$
S(f):=\left(\sum_{n \in \mathbb{N}^{d}}\left|d_{n} f\right|^{2}\right)^{1 / 2}, \quad s(f):=\left(\sum_{n \in \mathbb{N}^{d}} E_{n-1}\left|d_{n} f\right|^{2}\right)^{1 / 2},
$$

respectively, where the martingale differences are given with

$$
d_{n} f=\sum_{\epsilon_{i} \in\{0,1\}}(-1)^{\epsilon_{1}+\cdots+\epsilon_{d}} f_{n_{1}-\epsilon_{1}, \ldots, n_{d}-\epsilon_{d}} .
$$

For $0<p, q \leq \infty$ the martingale Hardy-Lorentz spaces $H_{p, q}^{S}$ and $H_{p, q}^{s}$ consist of all $d$-parameter martingales for which

$$
\|f\|_{H_{p, q}^{S}}:=\|S(f)\|_{p, q}<\infty, \quad\|f\|_{H_{p, q}^{s}}:=\|s(f)\|_{p, q}<\infty
$$

respectively. It is known that $H_{p, q}^{S} \sim L_{p, q}$ for $1<p<\infty$ and $0<q \leq \infty$.

In this paper the constants $C$ and $C_{p}$ may vary from line to line and the constants $C_{p}$ are depending only on $p$.

\section{Atomic decomposition of multi-parameter Hardy spaces}

Lemma 1 Let $0<p<\infty, 0<q \leq \infty,\left(2^{k} \eta_{k}\right) \in \ell_{q}, \eta_{k} \geq 0$ and $f \geq 0$. Suppose that there exists $0<\delta<1<\epsilon<\infty$ such that for all $N \in \mathbb{Z}, f \leq g_{N}+h_{N}$,

$$
P\left(g_{N}>2^{N}\right) \leq C 2^{-N \epsilon p} \sum_{k=-\infty}^{N} 2^{k \epsilon p} \eta_{k}^{p}
$$


and

$$
P\left(h_{N}>2^{N}\right) \leq C 2^{-N \delta p} \sum_{k=N}^{\infty} 2^{k \delta p} \eta_{k}^{p}
$$

Then $f \in L_{p, q}$ and

$$
\|f\|_{p, q} \leq C\left\|\left(2^{k} \eta_{k}\right)\right\|_{\ell_{q}} .
$$

Proof. It is enough to prove that

$$
\left\|\left(2^{k} P\left(f>2 \cdot 2^{k}\right)^{1 / p}\right)\right\|_{\ell_{q}} \leq C\left\|\left(2^{k} \eta_{k}\right)\right\|_{\ell_{q}} .
$$

It is easy to see that

$$
\left\|\left(2^{N} P\left(f>2 \cdot 2^{N}\right)^{1 / p}\right)\right\|_{\ell_{q}} \leq C\left\|\left(2^{N} P\left(g_{N}>2^{N}\right)^{1 / p}\right)\right\|_{\ell_{q}}+C\left\|\left(2^{N} P\left(h_{N}>2^{N}\right)^{1 / p}\right)\right\|_{\ell_{q}} .
$$

The inequality

$$
\left\|\left(2^{N} P\left(h_{N}>2^{N}\right)^{1 / p}\right)\right\|_{\ell_{q}} \leq C\left\|\left(2^{k} \eta_{k}\right)\right\|_{\ell_{q}}
$$

was proved in [1] and [7]. If $q=\infty$, then

$$
2^{N} P\left(g_{N}>2^{N}\right)^{1 / p} \leq C_{p} 2^{N-N \epsilon}\left(\sum_{k=-\infty}^{N} 2^{k(\epsilon-1) p} 2^{k p} \eta_{k}^{p}\right)^{1 / p} \leq C_{p}\left\|\left(2^{k} \eta_{k}\right)\right\|_{\ell_{\infty}},
$$

which proves the result. If $0<q<\infty$ and $p<q$, then apply Hölder's inequality with the exponent $r=q / p$ and its conjugate $r^{\prime}$ to obtain

$$
\begin{aligned}
2^{N} P\left(g_{N}>2^{N}\right)^{1 / p} & \leq C_{p} 2^{N-N \epsilon}\left(\sum_{k=-\infty}^{N} 2^{k \gamma p} 2^{k(\epsilon-\gamma) p} \eta_{k}^{p}\right)^{1 / p} \\
& \leq C_{p} 2^{N(1-\epsilon)}\left(\sum_{k=-\infty}^{N} 2^{k \gamma p r^{\prime}}\right)^{1 / p r^{\prime}}\left(\sum_{k=-\infty}^{N} 2^{k(\epsilon-\gamma) q} \eta_{k}^{q}\right)^{1 / q} \\
& \leq C_{p} 2^{N(1-\epsilon+\gamma)}\left(\sum_{k=-\infty}^{N} 2^{k(\epsilon-\gamma) q} \eta_{k}^{q}\right)^{1 / q}
\end{aligned}
$$

where $\gamma>0$ is arbitrary. If $p \geq q$, then we obtain the same inequality as follows:

$$
\begin{aligned}
2^{N} P\left(g_{N}>2^{N}\right)^{1 / p} & \leq C_{p} 2^{N(1-\epsilon+\gamma)}\left(\sum_{k=-\infty}^{N} 2^{k(\epsilon-\gamma) p} \eta_{k}^{p}\right)^{1 / p} \\
& \leq C_{p} 2^{N(1-\epsilon+\gamma)}\left(\sum_{k=-\infty}^{N} 2^{k(\epsilon-\gamma) q} \eta_{k}^{q}\right)^{1 / q}
\end{aligned}
$$


Hence

$$
\begin{aligned}
\left\|\left(2^{N} P\left(g_{N}>2^{N}\right)^{1 / p}\right)\right\|_{\ell_{q}}^{q} & \leq C_{p} \sum_{N \in \mathbb{Z}} 2^{N(1-\epsilon+\gamma) q} \sum_{k=-\infty}^{N} 2^{k(\epsilon-\gamma) q} \eta_{k}^{q} \\
& \leq C_{p} \sum_{k \in \mathbb{Z}} 2^{k(\epsilon-\gamma) q} \eta_{k}^{q} \sum_{N=k}^{\infty} 2^{N(1-\epsilon+\gamma) q} \\
& \leq C_{p} \sum_{k \in \mathbb{Z}} 2^{k} \eta_{k}^{q}
\end{aligned}
$$

whenever we choose $\gamma$ such that $0<\gamma<\epsilon-1$. The lemma is proved.

The atomic decomposition for $H_{p, q}^{s}$ is much more complicated in the multi-parameter setting. In this case, instead of the $\infty$-norm of the atoms, we have to use the 2-norm. The next theorem generalizes the atomic decomposition of $H_{p}^{s}$ (see Weisz [10]). We [10] generalized the stopping times for the multi-parameter setting as follows. A function $\tau$ which maps $\Omega$ into the set of subspaces of $\mathbb{N}^{d} \cup\{\infty\}$ is said to be a stopping time relative to $\left(\mathcal{F}_{n}, n \in \mathbb{N}^{d}\right)$ if the elements of $\tau(\omega)$ are incomparable for all $\omega \in \Omega$ and

$$
\{n \in \tau\} \in \mathcal{F}_{n} \quad\left(n \in \mathbb{N}^{d}\right) .
$$

The set of stopping times will be denoted by $\mathcal{T}$. A function $a \in L_{2}$ is called a $p$-atom if there exists a stopping time $\tau$ such that

(i) $a_{n}:=E_{n} a=0$ if $\tau \nless n$

(ii) $\left\|a^{*}\right\|_{2} \leq P(\tau \neq \infty)^{1 / 2-1 / p}(0<p<2)$.

Theorem $1 A$ d-parameter martingale $f$ is in $H_{p, q}^{s}(0<p<2,0<q \leq \infty)$ if and only if there exists a sequence $\left(a^{k}, k \in \mathbb{Z}\right)$ of p-atoms with associated stopping times $\left(\tau_{k}, k \in \mathbb{Z}\right)$ such that

$$
\left(\sum_{k \in \mathbb{Z}}\left|\mu_{k}\right|^{q}\right)^{1 / q}<\infty \quad \text { and } \quad \sum_{k \in \mathbb{Z}} \mu_{k} a_{n}^{k}=f_{n} \quad \text { a.e. }\left(n \in \mathbb{N}^{d}\right),
$$

where $\mu_{k}=\sqrt{2} \cdot 2^{k+1} P\left(\tau_{k} \neq \infty\right)^{1 / p}$. Moreover,

$$
\|f\|_{H_{p, q}^{s}} \sim \inf \left(\sum_{k \in \mathbb{Z}}\left|\mu_{k}\right|^{q}\right)^{1 / q}
$$

where the infimum is taken over all decompositions of $f$ of the form (3).

Proof. Assume that $f \in H_{p, q}^{s}$. Here we have to use finer stopping times than in the one-parameter case. Let

$$
\tau_{k}:=\inf \left\{n \in \mathbb{N}^{d}: E_{n} 1_{\left\{s(f)>2^{k}\right\}}>1 / 2\right\} .
$$


It is easy to see that

$$
f_{n}=\sum_{k \in \mathbb{Z}}\left(f_{n}^{\tau_{k+1}}-f_{n}^{\tau_{k}}\right)=\sum_{k \in \mathbb{Z}} \mu_{k} a_{n}^{k} \quad \text { a.e. } \quad\left(n \in \mathbb{N}^{d}\right)
$$

where

$$
a_{n}^{k}:=\frac{f_{n}^{\tau_{k+1}}-f_{n}^{\tau_{k}}}{\mu_{k}} .
$$

For a fixed $k,\left(a_{n}^{k}\right)$ is a martingale. In [10] we have shown that $a^{k}$ is a $p$-atom. For the sake of completeness, we give a short proof here. If $\tau_{k} \nless n$, then obviously $f_{n}^{\tau_{k+1}}=f_{n}^{\tau_{k}}$, thus (i) holds. Since $L_{2}$ is isometric to $H_{2}^{s}$, for (ii) we get that

$$
E\left(f_{n}^{\tau_{k+1}}-f_{n}^{\tau_{k}}\right)^{2} \leq E\left(\sum_{n \in \mathbb{N}^{d}} E_{n-1}\left|d_{n} f\right|^{2} 1_{\left\{\tau_{k} \ll n \ngtr \tau_{k+1}\right\}}\right)=(A)+(B),
$$

where

$$
(A)=\sum_{n \in \mathbb{N}^{d}} E\left(E_{n-1}\left|d_{n} f\right|^{2} 1_{\left\{\tau_{k} \ll n \ngtr \tau_{k+1}\right\}} 1_{\left\{s(f) \leq 2^{k+1}\right\}}\right)
$$

and

$$
(B)=\sum_{n \in \mathbb{N}^{d}} E\left(E_{n-1}\left|d_{n} f\right|^{2} 1_{\left\{\tau_{k} \ll n \ngtr \tau_{k+1}\right\}} 1_{\left\{s(f)>2^{k+1}\right\}}\right) .
$$

Clearly,

$$
(A) \leq 4^{k+1} P\left(\tau_{k} \neq \infty\right) .
$$

It follows from the definition of $\tau_{k+1}$ that if $\tau_{k+1} \nless n$, then

$$
E_{n-1} 1_{\left\{s(f)>2^{k+1}\right\}} \leq 1 / 2 .
$$

Hence

$$
\begin{aligned}
(B) & =\sum_{n \in \mathbb{N}^{d}} E\left(E_{n-1}\left|d_{n} f\right|^{2} 1_{\left\{\tau_{k} \ll n \ngtr \tau_{k+1}\right\}} E_{n-1} 1_{\left\{s(f)>2^{k+1}\right\}}\right) \\
& \leq \frac{1}{2} E\left(\sum_{n \in \mathbb{N}^{d}} E_{n-1}\left|d_{n} f\right|^{2} 1_{\left\{\tau_{k} \ll n \ngtr \tau_{k+1}\right\}}\right),
\end{aligned}
$$

which implies

$$
E\left(f_{n}^{\tau_{k+1}}-f_{n}^{\tau_{k}}\right)^{2} \leq 2 \cdot 4^{k+1} P\left(\tau_{k} \neq \infty\right)
$$

Thus

$$
E\left(\left(a_{n}^{k}\right)^{2}\right) \leq P\left(\tau_{k} \neq \infty\right)^{1-2 / p} \quad\left(n \in \mathbb{N}^{d}\right) .
$$

Hence there exists a function $a^{k} \in L_{2}$ such that $E_{n} a^{k}=a_{n}^{k}\left(n \in \mathbb{N}^{d}\right)$ and (ii) holds.

Next we obtain

$$
\begin{aligned}
P\left(\tau_{k} \neq \infty\right) & =P\left(\sup _{n \in \mathbb{N}^{d}} E_{n} 1_{\left\{s(f)>2^{k}\right\}}>1 / 2\right) \\
& \leq 4 E\left(\sup _{n \in \mathbb{N}^{d}}\left(E_{n} 1_{\left\{s(f)>2^{k}\right\}}\right)^{2}\right) \leq C P\left(s(f)>2^{k}\right) \leq C P(s(f)>u),
\end{aligned}
$$


where $2^{k-1} \leq u<2^{k}$. If $0<q<\infty$, then

$$
\sum_{k \in \mathbb{Z}}\left|\mu_{k}\right|^{q} \leq C \sum_{k \in \mathbb{Z}} \int_{2^{k-1}}^{2^{k}}\left(u P(s(f)>u)^{1 / p}\right)^{q} \frac{d u}{u} \leq C\|f\|_{H_{p, q}^{s}}^{q} .
$$

If $q=\infty$, then

$$
\sup _{k \in \mathbb{Z}}\left|\mu_{k}\right| \leq C \sup _{k \in \mathbb{Z}} 2^{k} P\left(s(f)>2^{k}\right)^{1 / p} \leq C\|f\|_{H_{p, \infty}} .
$$

Conversely, if the martingale $f$ has the above decomposition, then for an arbitrary integer $N$ let

$$
f_{n}=\sum_{k=-\infty}^{\infty} \mu_{k} a_{n}^{k}=g_{n}+h_{n} \quad\left(n \in \mathbb{N}^{d}\right)
$$

where

$$
g_{n}:=\sum_{k=-\infty}^{N-1} \mu_{k} a_{n}^{k} \quad \text { and } \quad h_{n}:=\sum_{k=N}^{\infty} \mu_{k} a_{n}^{k} .
$$

Obviously, $s(f) \leq s(g)+s(h)$. Since for a fixed $m$ the sets $\left\{\nu_{k} \ll m \ngtr \nu_{k+1}\right\}$ are disjoint and

$$
\mu_{k} a_{n}^{k}=\sum_{m \leq n}\left(d_{m} f\right) 1_{\left\{\nu_{k} \ll m \ngtr \nu_{k+1}\right\}},
$$

we obtain

$$
\begin{aligned}
P\left(s(g)>2^{N}\right) & \leq 2^{-2 N}\|s(g)\|_{2}^{2}=2^{-2 N} \int_{\Omega}\left|\sum_{k=-\infty}^{N-1} \mu_{k} a^{k}\right|^{2} d P \\
& =2^{-2 N} \sum_{k=-\infty}^{N-1} \int_{\Omega}\left|\mu_{k} a^{k}\right|^{2} d P=C 2^{-2 N} \sum_{k=-\infty}^{N} 2^{2 k} P\left(\tau_{k} \neq \infty\right) .
\end{aligned}
$$

Choosing $\epsilon=2 / p>1$, we obtain (1). Moreover,

$$
P\left(s(h)>2^{N}\right) \leq P(s(h)>0) \leq \sum_{k=N}^{\infty} P\left(s\left(a^{k}\right)>0\right) \leq \sum_{k=N}^{\infty} P\left(\tau_{k} \neq \infty\right),
$$

which proves (2). By Lemma 1 we conclude that

$$
\|s(f)\|_{p, q} \leq C\left\|\left(2^{k} P\left(\tau_{k} \neq \infty\right)^{1 / p}\right)\right\|_{\ell_{q}}=C\left\|\left(\mu_{k}\right)\right\|_{\ell_{q}} .
$$

The proof of the theorem is complete.

If $\mathcal{F}$ is regular, then the previous theorem can be shown for the $H_{p, q}^{S}$ spaces as well. 
Theorem 2 Suppose that the stochastic basis $\mathcal{F}$ is regular. A d-parameter martingale $f$ is in $H_{p, q}^{S}(0<p<2,0<q \leq \infty)$ if and only if there exists a sequence $\left(a^{k}, k \in \mathbb{Z}\right)$ of p-atoms with associated stopping times $\left(\tau_{k}, k \in \mathbb{Z}\right)$ such that

$$
\left(\sum_{k \in \mathbb{Z}}\left|\mu_{k}\right|^{q}\right)^{1 / q}<\infty \quad \text { and } \quad \sum_{k \in \mathbb{Z}} \mu_{k} a_{n}^{k}=f_{n} \quad \text { a.e. }\left(n \in \mathbb{N}^{d}\right),
$$

where $\mu_{k}=\sqrt{2} \cdot 2^{k+1} P\left(\tau_{k} \neq \infty\right)^{1 / p}$. Moreover,

$$
\|f\|_{H_{p, q}^{S}} \sim \inf \left(\sum_{k \in \mathbb{Z}}\left|\mu_{k}\right|^{q}\right)^{1 / q}
$$

where the infimum is taken over all decompositions of $f$ of the form (4).

Corollary 1 If $\mathcal{F}$ is regular, then $H_{p, q}^{s} \sim H_{p, q}^{S}$ for all $0<p<2,0<q \leq \infty$.

Note that this corollary was already proved in [10] with another method.

\section{Duality theorems}

In [10] we have introduced the $B M O_{r}(\alpha)$ space (there it was denoted by $\Lambda_{r}(\alpha)$ ) and proved that the dual of $H_{p}^{s}$ is $B M O_{2}(\alpha)$ with $0<p \leq 1, \alpha=1 / p-1$. $B M O_{r}(\alpha)$ $(1 \leq r<\infty, \alpha>-1 / r)$ denotes the space of those functions $f \in L_{r}$ for which

$$
\|f\|_{B M O_{r}(\alpha)}=\sup _{\tau \in \mathcal{T}} P(\tau \neq \infty)^{-1 / r-\alpha}\left\|f-f^{\tau}\right\|_{r}<\infty .
$$

We generalize these spaces as follows. A functions $f \in L_{r}$ is in $B M O_{r, q}(\alpha)(1 \leq r<$ $\infty, \alpha>-1 / r, 0<q<\infty)$ if

$$
\|f\|_{B M O_{r, q}(\alpha)}=\sup _{\tau_{k} \in \mathcal{T}} \frac{\sum_{k \in \mathbb{Z}} 2^{k} P\left(\tau_{k} \neq \infty\right)^{1-1 / r}\left\|f-f^{\tau_{k}}\right\|_{r}}{\left(\sum_{k \in \mathbb{Z}}\left(2^{k} P\left(\tau_{k} \neq \infty\right)^{1+\alpha}\right)^{q}\right)^{1 / q}}<\infty,
$$

where the supremum is taken over all stopping times $\tau_{k}$, for which $\left(2^{k} P\left(\tau_{k} \neq \infty\right)^{1+\alpha}\right) \in$ $\ell_{q}$. If we take only one stopping time in the supremum of the $B M O_{r, q}(\alpha)$-norm, then we get back the $B M O_{r}(\alpha)$-norm, i.e., $\|f\|_{B M O_{r}(\alpha)} \leq\|f\|_{B M O_{r, q}(\alpha)}$. On the other hand, if $0<q \leq 1$, then

$$
\|f\|_{B M O_{r, q}(\alpha)} \leq \sup _{\tau_{k} \in \mathcal{T}} \frac{\sum_{k \in \mathbb{Z}} 2^{k} P\left(\tau_{k} \neq \infty\right)^{1+\alpha}\|f\|_{B M O_{r}(\alpha)}}{\left(\sum_{k \in \mathbb{Z}}\left(2^{k} P\left(\tau_{k} \neq \infty\right)^{1+\alpha}\right)^{q}\right)^{1 / q}} \leq\|f\|_{B M O_{r}(\alpha)},
$$

so in this case $B M O_{r}(\alpha) \sim B M O_{r, q}(\alpha)$. In case $\alpha=0$, we denote the spaces by $B M O_{r}$ and $B M O_{r, q}$.

These spaces were first introduced and investigated in the one-parameter case by Jiao, Xie and Zhou [7] (see also Ho [5]). They proved the one-parameter version of Theorems 3-6. Since the following theorems can be shown similarly as in the oneparameter case (see [7]), we omit the proofs. 
Theorem 3 The dual space of $H_{p, q}^{s}$ is $B M O_{2}(\alpha),(0<p<2,0<q \leq 1, \alpha=1 / p-1)$.

Theorem 4 The dual space of $H_{p, q}^{s}$ is $B M O_{2, q}(\alpha),(0<p<2,1<q<\infty, \alpha=$ $1 / p-1)$.

Theorem 5 If $\mathcal{F}$ is regular, then the dual space of $H_{p, q}^{s}$ is $B M O_{r}(\alpha),\left(0<p<r^{\prime} \leq\right.$ $2,0<q \leq 1, \alpha=1 / p-1)$.

Theorem 6 If $\mathcal{F}$ is regular, then the dual space of $H_{p, q}^{s}$ is $B M O_{r, q}(\alpha),\left(0<p<r^{\prime} \leq\right.$ $2,1<q<\infty, \alpha=1 / p-1)$.

Corollary 2 Suppose that $\mathcal{F}$ is regular, $2 \leq r<\infty, 1<q<\infty$ and $\alpha>-1 / r$. Then $\mathrm{BMO}_{2}(\alpha)$ is equivalent to $B M O_{r}(\alpha)$ and $B M O_{2, q}(\alpha)$ is equivalent to $B M O_{r, q}(\alpha)$.

Note that the first half of Corollary 2 was proved in [10] for $\alpha=0$, i.e., $B M O_{2} \sim$ $B M O_{r}(2 \leq r<\infty)$.

\section{References}

[1] W. Abu-Shammala and A. Torchinsky. The Hardy-Lorentz Spaces $H^{p, q}\left(R^{n}\right)$. Studia Math., 182:283-294, 2007.

[2] C. Fefferman and E. M. Stein. $H^{p}$ spaces of several variables. Acta Math., 129:137$194,1972$.

[3] A. M. Garsia. Martingale Inequalities. Seminar Notes on Recent Progress. Math. Lecture Note. Benjamin, New York, 1973.

[4] L. Grafakos. Classical and Modern Fourier Analysis. Pearson Education, New Jersey, 2004.

[5] K.-P. Ho. Atomic decompositions, dual spaces and interpolations of martingale Hardy-Lorentz-Karamata spaces. Quart. J. Math., 65:985-1009, 2014.

[6] Y. Jiao, L. Peng, and P. Liu. Atomic decompositions of Lorentz martingale spaces and applications. J. Funct. Spaces Appl., 7(2):153-166, 2009.

[7] Y. Jiao, G. Xie, and D. Zhou. Dual spaces and John-Nirenberg inequalities of martingale Hardy-Lorentz-Karamata spaces. Quart. J. Math., 66:605-623, 2015.

[8] R. Long. Martingale Spaces and Inequalities. Peking University Press and Vieweg Publishing, 1993.

[9] Y. Ren. The dual spaces of martingale Hardy-Lorentz spaces. Publ. Math. Debrecen, 86:457-463, 2015.

[10] F. Weisz. Martingale Hardy Spaces and their Applications in Fourier Analysis, volume 1568 of Lecture Notes in Math. Springer, Berlin, 1994.

[11] F. Weisz. Summability of Multi-dimensional Fourier Series and Hardy Spaces. Mathematics and Its Applications. Kluwer Academic Publishers, Dordrecht, Boston, London, 2002. 